\title{
A randomised controlled trial of multiple periods of outdoor free-play to increase moderate-to-vigorous physical activity among 3 to 6 year old children attending childcare: study protocol
}

Luke Wolfenden ${ }^{1,2,3,4^{*}}$, John Wiggers ${ }^{1,2,3,4}$, Philip Morgan ${ }^{5}$, Lubna Abdul Razak¹,2,3, Jannah Jones 1,2,3,4, Meghan Finch ${ }^{1,2,3}$, Rachel Sutherland ${ }^{1,2,3}$, Christophe Lecathelinais ${ }^{1}$, Karen Gillham ${ }^{1}$ and Sze Lin Yoong ${ }^{1,2,3,4}$

\begin{abstract}
Background: The implementation of physical activity interventions in centre-based childcare services has been recommended to improve child health. This study aims to evaluate the efficacy of scheduling multiple periods of outdoor free play in increasing the time children spend in moderate-to-vigorous physical activity (MVPA) during childcare.

Methods: The study will employ a between group cluster randomised controlled trial design. Fourteen childcare services in the Hunter New England region of New South Wales, Australia, who currently implement a single session of free outdoor play between their core operational hours of 9 am to $3 \mathrm{pm}$ will be recruited into the trial. Childcare services will be randomised to an intervention or a no intervention control group. Childcare services in the intervention group will be supported by an early childhood education specialist to provide three periods of outdoor free play for children between the hours of 9 am to $3 \mathrm{pm}$. Each period of outdoor free play will be at least $15 \mathrm{~min}$ in duration but must equate to their total usual duration of outdoor play. Services in the control group will continue to implement a single period of outdoor play. The primary trial outcome is minutes of time children spend in MVPA whilst in care assessed objectively via accelerometer over 5 days. Outcome assessment will occur at baseline and 3 months post baseline. Generalised Linear Mixed Models (GLMM) under an intention to treat framework will be used to compare differences between groups in the primary trial outcome at follow-up. Sensitivity analysis will be conducted to test assumptions of missing data. Per protocol analysis will be performed using services that implemented the intervention as intended and subgroup analysis undertaken by gender and baseline physical activity levels of children.
\end{abstract}

Discussion: The study tests a simple ecological intervention that has the potential to increase child physical activity in care. Trial registration: Australian New Zealand Clinical Trials Registry 12616000347460. Prospectively registered 17th March 2016.

Keywords: Physical activity, Randomised controlled trial, Intervention, Childcare, Preschool, Outdoor play, Activity breaks

\footnotetext{
* Correspondence: luke.wolfenden@hnehealth.nsw.gov.au

${ }^{1}$ Hunter New England Population Health, Locked Bag 10, Wallsend, NSW

2287, Australia

${ }^{2}$ School of Medicine and Public Health, University of Newcastle, Callaghan,

NSW 2308, Australia

Full list of author information is available at the end of the article
}

\section{$\int$ Biomed Central}

(c) 2016 The Author(s). Open Access This article is distributed under the terms of the Creative Commons Attribution 4.0 International License (http://creativecommons.org/licenses/by/4.0/), which permits unrestricted use, distribution, and reproduction in any medium, provided you give appropriate credit to the original author(s) and the source, provide a link to the Creative Commons license, and indicate if changes were made. The Creative Commons Public Domain Dedication waiver (http://creativecommons.org/publicdomain/zero/1.0/) applies to the data made available in this article, unless otherwise stated. 


\section{Background}

Inadequate physical activity is associated with the most prevalent causes of mortality and morbidity including obesity, diabetes, cardiovascular disease and some cancers [1]. Physical activity in early childhood has immediate beneficial effects on blood pressure, lipid profile, motor skill and bone development [2-4] with greatest benefit accruing at moderate-to-vigorous intensity [3, 4]. Despite these benefits, research in Australia and internationally has demonstrated that most children aged 2 to 6 years do not engage in physical activity consistent with current national guidelines $[5,6]$.

A number of characteristics of centre-based childcare services suggest that they represent an ideal setting for interventions to improve physical activity in young children. First, centre-based childcare provides access to a significant proportion of the population aged less than 5 years, often for prolonged periods. For example, approximately $55 \%$ of Australian children aged 0 to 5 years attended some form of centre-based care in 2014 [7]. Second, reviews suggest that young children are not sufficiently active during attendance at centre-based care, necessitating interventions in this setting [8]. Third, childcare service staff believe in the importance of children being physically active as a part of their professional responsibility and are amenable to interventions to support improvements in child activity [9].

Modifying the frequency of outdoor free-play may represent a potentially innovative and effective strategy in improving the physical activity of children attending childcare. Unstructured outdoor free-play (as opposed to structured, staff-guided play) has been consistently associated with greater child physical activity among children in care $[10,11]$. However, findings of a randomised trial suggest that increasing the duration of time that children in childcare have available for outdoor free-play may not be effective in improving child physical activity [12]. A likely explanation for this is that extending outdoor free-play time alone does not account for children's natural physical activity patterns. Children's activity in care is characterised by short, intense bouts of activity of between 3 and 15 min occurring at the start of outdoor free-play opportunities, followed by extended recovery periods of sedentary behaviour or light activity [13-16]. As such, extending periods of outdoor play alone may not increase child moderate-to-vigorous physical activity (MVPA). However, even if the total duration of outdoor free-play time for children remains constant, scheduling shorter but more frequent opportunities for outdoor free-play may enhance child physical activity by promoting more spontaneous bouts of intense activity ('activity peaks') that is characteristic of the first $15 \mathrm{~min}$ of outdoor free-play [13].

In this context the aim of this study is to assess the efficacy of scheduling multiple periods of outdoor free- play per day in increasing the time children spend in MVPA during childcare relative to one outdoor play time of the same duration. This manuscript describes the trial methods and trial outcomes of the study.

\section{Methods \\ Design}

The study will employ a between group, cluster randomised controlled trial design (see Fig. 1). Fourteen centre-based childcare services with one period of outdoor free-play (of at least $45 \mathrm{~min}$ duration) will be randomised to an intervention or control group. Intervention services will change their scheduling of outdoor free-play such that their usual total time for outdoor free-play is broken into multiple separate periods of at least $15 \mathrm{~min}$ in duration each with an indoor period of at least $30 \mathrm{~min}$ in between. Control services will continue to provide their usual total period of outdoor freeplay time across a single continuous period. Intervention efficacy will be determined by comparing differences between groups in the minutes children spend in MVPA per day at childcare. MVPA will be assessed via accelerometer over 5 days at baseline and 3 months later. Approval to conduct the study was obtained from Hunter New England Human Research Ethics Committee (reference number 15/11/18/4.03) and the University of

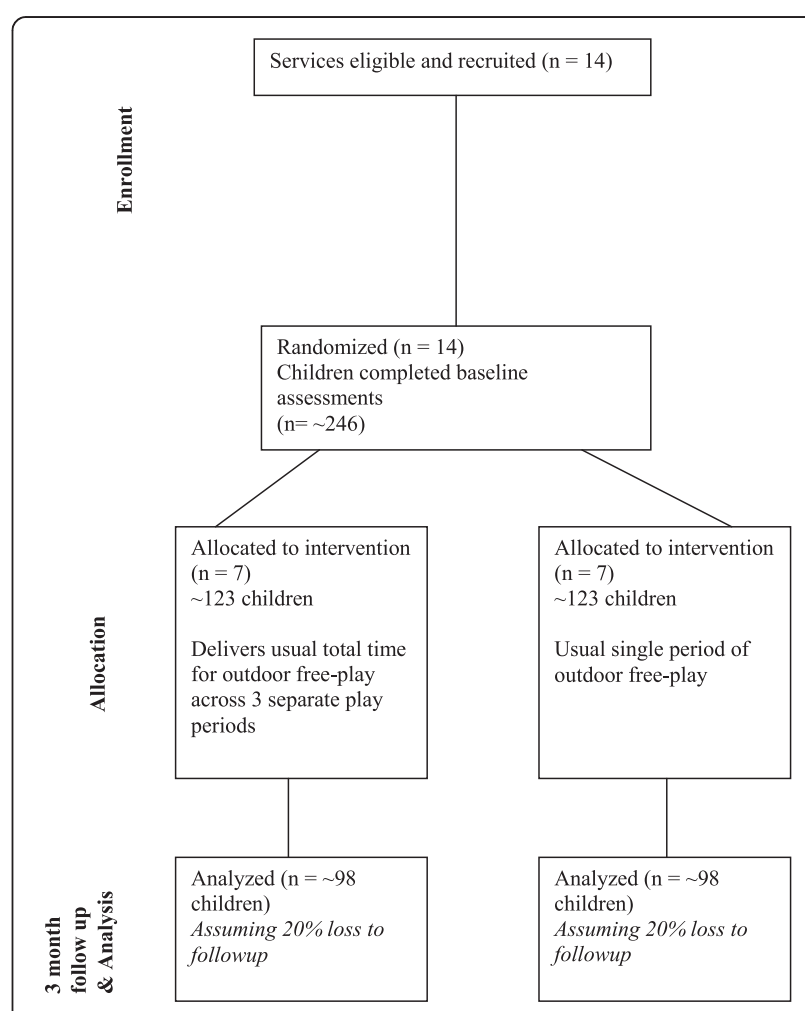

Fig. 1 Flow of participants through each stage 
Newcastle Human Research Ethics Committee (reference number H-2016-0088).

\section{Participant eligibility and evidence-based recruitment strategy \\ Childcare services}

To be eligible to participate in the trial, centre-based childcare services (defined as long day care services or preschools) will be required to have an enrolment of least 25 children aged between 3 to 6 years, be located within the Hunter or New England regions of New South Wales, Australia, and have only one outdoor freeplay session occurring between the core operational hours of 9 am to $3 \mathrm{pm}$. Childcare services catering solely for special needs populations, or those participating in other physical activity interventions will be excluded from participating in the trial.

A list of all childcare services that are licensed to provide care for 3 to 6 year-old children located within the study region will be obtained from the licensing agency. Supervisors of childcare services across the study region will be sent study information prior to telephone contact to assess eligibility and to invite study participation among eligible services. Recruitment will continue until 14 services have consented to participate. Previous studies in childcare utilising this recruitment approach have yielded a childcare service participation rate of $81-84 \%$ [17-19].

\section{Children}

Active parental consent will be required for study participation. To be eligible for the data collection component of the study, children will be required to be aged 3 to 6 years and attend participating services between 9 am and $3 \mathrm{pm}$ on one or more days in the week of data collection. Children with an intellectual or physical impairment that may impact on their physical activity capacity or prevent them from complying with data collection protocols will be excluded.

To maximise study participation, children will be recruited utilising an evidenced-based strategy recommended for obtaining active parental consent for child participation in school-based research, which will include a mail out of study information and consent forms prior to onsite recruitment, face to face dissemination of information sheets and provision of consent forms to parents during periods of drop-off or pick-up from childcare [20]. Such a recruitment strategy has been utilised in previous trials of interventions for preschool aged children in this setting [21-23].

\section{Randomisation, allocation and blinding}

A statistician with no other involvement in recruitment or data collection will allocate services to either the intervention or control condition in a 1:1 ratio using a computerised random number generator. Randomisation of childcare services will be stratified by the socioeconomic status of the area where the service is located, and on the service type (long day care service or preschool) based on evidence of an association between these factors and the physical activity policies and practices of services [24].

\section{Intervention}

The intervention will seek to create a childcare environment more supportive of child physical activity by scheduling multiple opportunities for outdoor free-play in a way which is consistent with children's developing fitness levels [25] and natural physical activity patterns $[10,13,26]$. Specifically, within a 6 h day (9 am to $3 \mathrm{pm})$ the intervention will divide their usual total time of outdoor free-play for children across three periods of at least 15 min duration.

Three periods of outdoor free-play was selected as: i) research has demonstrated repeated spikes in activity across the day in childcare coinciding with scheduled breaks $[13,16]$; and ii) that the start of such breaks stimulate between 3 and 15 min of intense activity, sufficient to achieve an additional 10 min MVPA [27]. The minimum period of outdoor free-play of $15 \mathrm{~min}$ was selected given evidence that MVPA often attenuates after this period [13]. Furthermore, services will be required to schedule at least $30 \mathrm{~min}$ of indoor time (structured or unstructured play) between periods of outdoor free-play, based on evidence that children may be more likely to be moderate-to-vigorously physically active following prolonged indoor periods [28]. Immediately following baseline data collection, intervention services will be supported by a member of the research team and an early childhood education specialist to implement the intervention. This support will include written materials and at least two site visits and two telephone support calls prior to follow-up data collection to re-orient their operations to incorporate the scheduling change. No other intervention support will be provided to children, childcare service staff or parents.

\section{Control}

Services allocated to the control group will schedule their usual single period of outdoor free-play for children across the day. Control services will also be instructed to continue the one outdoor free-play period of the same duration (except during inclement weather) across the study period.

\section{Data collection and measures}

Data will be collected at baseline and approximately 3 months post baseline. 


\section{Primary trial outcome-minutes spent in moderate-to- vigorous physical activity in care}

The primary trial outcome is the number of minutes children spend in MVPA during the core hours of service operation (9 am to $3 \mathrm{pm}$ ). MVPA will be objectively assessed using an Actigraph GT3X+ accelerometer using recommended cut-points [29]. The Actigraph accelerometer has established utility, validity and reliability and is the current gold standard for assessment of activity in children aged 3 to 6 years old [30].

Accelerometers will be worn by children during the core operational hours of childcare ( 9 am to $3 \mathrm{pm})$. Two data collectors, not blinded to allocation at follow-up, will attend services to fit and collect accelerometers using a standard protocol [29]. Accelerometers will be placed above the iliac crest at the hip of the child using a clip or band. Accelerometer data will be collected on every day of 1 week (5 days in total) of the data collection period at baseline and follow-up. Children will wear the accelerometer each day (up to 5 days) that they attend care. The accelerometer will be fitted as the children arrive at the childcare service and removed at $3 \mathrm{pm}$ or earlier when the child departs the service. Despite evidence to suggest that increases in child activity at childcare do not result in decreased activity at home [31], at baseline and follow-up, consent will be sought for children to wear accelerometers during 'out of care' hours also to assess potential compensatory effects.

\section{Secondary trial outcomes}

Secondary trial outcomes include total child activity (counts per minute collected in $5 \mathrm{~s}$ epochs) in care [32] and percent of time children spend in MVPA adjusted for wear time, assessed via accelerometer. Additionally, as a potential adverse effect of the intervention, during interviews with childcare services, the number of injuries requiring documentation will be assessed using items taken from previous childcare physical activity studies [21].

\section{Child and parent characteristics}

A computer-assisted telephone interview with parents will be conducted to collect: child and parent demographic information (age, gender, household income, and parent education); usual parent physical activity; and child and parent height and weight, using items from the New South Wales Population Health Survey [33] and items to assess the home environment from the preschool age physical activity questionnaire (Pre-PAQ) questionnaire [34]. The survey will be conducted at baseline and follow-up.

\section{Service characteristics}

During a telephone interview with supervisors of participating childcare services the following service characteristics will be assessed: postcode of locality (to assess the socioeconomic status of the area) [35]; number of years in operation; total number of 3 to 6 year-old children enrolled; number of staff; staff qualifications; and service governance (Department of Education service or privately owned). Such items were drawn from previous studies [17, 24].

\section{Service outdoor free-play schedule and physical activity environment}

Observations at childcare services will be conducted by data collectors (not blind to group allocation) to record the duration (via stop watch), timing and frequency of outdoor free-play to ensure that services are implementing outdoor free-play periods consistent with the study protocol. Data collectors will also collect information regarding the childcare service physical activity environment using a comprehensive environment assessment tool (Environment and Policy Assessment and Observation instrument, EPAO) [36]. The following types of physical activity observation elements will be conducted: Active play opportunities, sedentary opportunities, sedentary environment, portable play environment, fixed play environment, staff behaviour physical activity, physical activity training and education and physical activity policy.

\section{Intervention fidelity}

The research team will visit the services during the intervention period to observe if childcare services have implemented the intervention prior to follow-up data collection. A checklist developed by the research team will be used to monitor whether the intervention was delivered as per the study protocol on the day of data collection.

\section{Analysis}

Minutes of MVPA will be determined using age-specific child-validated equations (cut points) [29]. Generalised Linear Mixed Models (GLMM), to take account of the repeated measures on children (daily measures during baseline and follow-up periods) as well as clustering of individuals within services, will be used under an intention to treat framework to test for a difference in change in minutes of MVPA between groups. The GLMM will include terms for time (baseline and followup), group (intervention or control group), and the interaction of time and group, and will control for child gender and total outdoor play time. A sensitivity analysis will be performed using multiple imputation for missing data to assess robustness of the main analysis [37]. Per protocol analysis will be performed using services that implemented the intervention as intended and subgroup analysis undertaken by gender and baseline physical activity levels of children. 


\section{Sample size and power calculation}

The study will approach approximately 500 children from 14 childcare services across the study region. Assuming the standard deviation of MVPA is $2.7 \mathrm{~min} / \mathrm{h}$ [38] and assuming an intraclass correlation coefficient of 0.1 [39], a sample of 14 children per cluster (assuming a conservative participation rate of approximately 50 and a $20 \%$ loss to follow-up) will provide the study with $80 \%$ power to detect a change of 9.9 min in MVPA. An increase of $10 \mathrm{~min}$ of MVPA in children aged 3 to 6 years old has been found to have clinically significant beneficial effects on fat mass and peak bone mass $[3,4]$.

\section{Discussion}

Supporting physical activity in early childhood is a recommended strategy [40] to reduce the community health burden of inactivity, as physical activity in childhood persists over time [41], and health behaviours in childhood are more easily influenced than behaviours in adolescents and adults [42]. While previous observational studies have reported that children are frequently sedentary or engaged in light activity, in recent years has research began to accumulate that describes patterns of activity among children aged 3 to 6 years old across a day in childcare $[10,13,16]$. Through the use of experimental methods, the trial will provide rigorous evidence to indicate whether interventions sensitive to child activity patterns by increasing the frequency of bouts of outdoor free-play are effective. Even if moderately effective, the intervention has the potential to improve the health and wellbeing of the hundreds of thousands of Australian children who attend some form of centrebased childcare through reducing the risk of the precursors of chronic disease.

\section{Acknowledgements}

The authors thank participating childcare services.

\section{Funding}

This project is funded by the National Health and Medical Research Council (NHMRC) project grant number APP1083927. The content of this publication is the responsibility of the authors and do not reflect the views of NHMRC. Hunter New England Population Health and the University of Newcastle also provided infrastructure funding.

\section{Availability of data and materials}

Study materials and data will be held by the Administering Institution. Deidentified data-sets and all study materials are available on request from the corresponding author.

\section{Authors' contributions}

LW, JW, PM conceived the study and secured funding. SY, LW, PM, JW, JJ, LAR designed the study procedures. LW led the drafting of the manuscript. All authors contributed to, read and approved the final version of the manuscript.

\section{Competing interests}

The authors declare that they have no competing interests.

\section{Consent for publication}

Not applicable.

\section{Ethics approval and consent to participate}

Ethical approval to conduct the study has been obtained from the Hunter New England Human Research Ethics Committee (reference number 15/11/ 18/4.03) and the University of Newcastle Human Research Ethics Committee (reference number $\mathrm{H}$-2016-0088). Active parental consent will be required for child participation in the study.

\section{Author details}

'Hunter New England Population Health, Locked Bag 10, Wallsend, NSW 2287, Australia. ${ }^{2}$ School of Medicine and Public Health, University of Newcastle, Callaghan, NSW 2308, Australia. ${ }^{3}$ Hunter Medical Research Institute, Newcastle, NSW 2300, Australia. ${ }^{4}$ Priority Research Centre for Health Behaviour, University of Newcastle, Callaghan, NSW 2308, Australia. ${ }^{5}$ School of Education, Priority Research Centre for Physical Activity and Nutrition, University of Newcastle, Callaghan, NSW 2308, Australia.

Received: 5 August 2016 Accepted: 27 August 2016

Published online: 02 September 2016

\section{References}

1. AlHW. Australia's Health 2014. Canberra: Australian Institute of Health and Welfare; 2014

2. Burgi F, Meyer U, Granacher U, Schindler C, Marques-Vidal P, Kriemler S, Puder JJ. Relationship of physical activity with motor skills, aerobic fitness and body fat in preschool children: a cross-sectional and longitudinal study (Ballabeina). Int J Obes (Lond). 2011;35(7):937-44.

3. Janz KF, Kwon S, Letuchy EM, Eichenberger Gilmore JM, Burns TL, Torner JC, Willing MC, Levy SM. Sustained effect of early physical activity on body fat mass in older children. Am J Prev Med. 2009;37(1):35-40.

4. Janz KF, Letuchy EM, Eichenberger Gilmore JM, Burns TL, Torner JC, Willing MC, Levy SM. Early physical activity provides sustained bone health benefits later in childhood. Med Sci Sports Exerc. 2010;42(6):1072-8.

5. Reilly JJ. Low levels of objectively measured physical activity in preschoolers in child care. Med Sci Sports Exerc. 2010;42(3):502-7.

6. Hnatiuk JA, Salmon J, Hinkley T, Okely AD, Trost S. A review of preschool children's physical activity and sedentary time using objective measures. Am J Prev Med. 2014;47(4):487-97.

7. Australian Bureau of Statistics A. Childhood Education and Care, Australia, June 2014. In: Cat no 44020DO6006 201406. Canberra: ABS; 2015.

8. Dowda M, Pate RR, Trost SG, Almeida M, Joao CA, Sirard JR. Influences of preschool policies and practices on children's physical activity. J Community Health. 2004;29(3):183-96.

9. Pagnini D, Wilkenfeld R, King L, Booth M, Booth S. Early childhood sector staff perceptions of child overweight and obesity: the Weight of Opinion Study. Health Promot J Austr. 2007;18(2):149-54.

10. Brown WH, Pfeiffer KA, Mclver KL, Dowda M, Addy CL, Pate RR. Social and environmental factors associated with preschoolers' nonsedentary physical activity. Child Dev. 2009;80(1):45-58.

11. Gordon ES, Tucker P, Burke SM, Carron AV. Effectiveness of physical activity interventions for preschoolers: a meta-analysis. Res Q Exerc Sport. 2013;84(3):287-94

12. Alhassan S, Sirard JR, Robinson TN. The effects of increasing outdoor play time on physical activity in Latino preschool children. Int J Pediatr Obes. 2007:2(3):153-8

13. Pate RR, Dowda M, Brown WH, Mitchell J, Addy C. Physical activity in preschool children with the transition to outdoors. J Phys Act Health. 2013;10:170-5.

14. Pate R, Mclver K, Dowda M, Brown WH, Addy C. Directly observed physical activity levels in preschool children. J School Health. 2008;78(8):438-44.

15. Timmons BW, Naylor P-J, Pfeiffer KA. Physical activity for preschool children how much and how? Appl Physiol Nutr Metab. 2007:32(Suppl 2F):S122-135.

16. Verbestel V, Cauwenberghe EV, Coen VD, Maes L, Bourdeaudhuij ID, Cardon $\mathrm{G}$. Within- and between-day variability of objectively measured physical activity in preschoolers. Pediatr Exerc Sci. 2011;23:366-78.

17. Finch M, Wolfenden L, Falkiner M, Edenden D, Pond N, Hardy LL, Milat AJ, Wiggers J. Impact of a population based intervention to increase the adoption of multiple physical activity practices in centre based childcare services: a quasi experimental, effectiveness study. Int J Behav Nutr Phys Act. 2012;9:101. 
18. Finch M, Wolfenden L, Morgan PJ, Freund M, Wyse R, Wiggers J. A cluster randomised trial to evaluate a physical activity intervention among 3-5 year old children attending long day care services: study protocol. BMC Public Health. 2010;10:534.

19. Jones J, Wyse R, Finch M, Lecathelinais C, Wiggers J, Marshall J, Falkiner M, Pond $\mathrm{N}$, Yoong SL, Hollis J, et al. Effectiveness of an intervention to facilitate the implementation of healthy eating and physical activity policies and practices in childcare services: a randomised controlled trial. Implement Sci. 2015;10:147.

20. Wolfenden L, Kypri K, Freund M, Hodder R. Obtaining active parental consent for school-based research: a guide for researchers. Aust N Z J Public Health. 2009;33(3):270-5.

21. Finch M, Wolfenden L, Morgan PJ, Freund M, Jones J, Wiggers J. A cluster randomized trial of a multi-level intervention, delivered by service staff, to increase physical activity of children attending center-based childcare. Prev Med. 2014:58:9-16.

22. Wyse R, Wolfenden L, Campbell E, Campbell KJ, Wiggers J, Brennan L, Fletcher A, Bowman J, Heard TR. A cluster randomized controlled trial of a telephone-based parent intervention to increase preschoolers' fruit and vegetable consumption. Am J Clin Nutr. 2012;96(1):102-10.

23. Wolfenden L, Wyse R, Campbell E, Brennan L, Campbell KJ, Fletcher A, Wiggers J, Bowman J, Heard TR. Randomized controlled trial of a telephone-based intervention for child fruit and vegetable intake: long-term follow-up. Am J Clin Nutr. 2014:99(3):543-50.

24. Wolfenden L, Neve M, Farrell L, Lecathelinais C, Bell C, Milat A, Wiggers J, Sutherland R. Physical activity policies and practices of childcare centres in Australia. J Paediatr Child Health. 2011:47(3):73-6.

25. Brown WH, Googe HS, Mclver KL, Rathel JM. Effects of teacher-encouraged physical activity on preschool playgrounds. J Early Intervent. 2009:31(2):126-45.

26. Kreichauf S, Wildgruber A, Krombholz H, Gibson EL, Vogele C, Nixon CA, Douthwaite W, Moore HJ, Manios Y, Summerbell CD, et al. Critical narrative review to identify educational strategies promoting physical activity in preschool. Obes Rev. 2012;13 Suppl 1:96-105.

27. Greever CJ, Sirard J, Alhassan S. Objective analysis of preschoolers' physical activity patterns during free playtime. J Phys Act Health. 2015;12(9):1253-8,

28. Smith PK, Hagan T. Effects of deprivation on exercise play in nursery school children. Anim Behav. 1980;28(3):922-8.

29. Cliff DP, Reilly JJ, Okely AD. Methodological considerations in using accelerometers to assess habitual physical activity in children aged 0-5 years. J Sci Med Sport. 2009;12(5):557-67.

30. Cain KL, Sallis JF, Conway TL, Dyck DV, Calhoon L. Using accelerometers in youth physical activity studies: a review of methods. J Phys Act Health. 2013;10:437-50.

31. Dale $D$, Corbin $C B$, Dale K. Restricting opportunities to be active during school time: do children compensate by increasing physical activity levels after school? Res Q Exerc Sport. 2000;71(3):240-8.

32. Vale S, Trost S, Ruiz JJ, Rego C, Moreira P, Mota J. Physical activity guidelines and preschooler's obesity status. Int J Obes (Lond). 2013;37(10):1352-5.

33. NSW Population Health Survey 2014 - Questionnaire [http://www.health. nsw.gov.au/surveys/adult/Documents/Questionnaire-2014-WEB.pdf]. Accessed 21 Jan 2016.

34. Dwyer GM, Hardy LL, Peat JK, Baur LA. The validity and reliability of a home environment preschool-age physical activity questionnaire (Pre-PAQ). Int J Behav Nutr Phys Act. 2011;8:86.

35. Australian Bureau of Statistics A. Census of Population and Housing: SocioEconomic Indexes for Areas (SEIFA), Australia 2011. In: Cat no: 2033055001. 2013.

36. Ward DS, Vaughn A, McWilliams C, Hales D. Interventions for increasing physical activity at child care. Med Sci Sports Exerc. 2010;42(3):526-34.

37. White IR, Horton NJ, Carpenter J, Pocock SJ. Strategy for intention to treat analysis in randomised trials with missing outcome data. BMJ. 2011;342:d40.

38. Pate RR, Brown WH, Pfeiffer KA, Howie EK, Saunders RP, Addy CL, Dowda M. An intervention to increase physical activity in children. Am J Prev Med. 2016; 51(1):12-22

39. Murray DM, Varnell SP, Blitstein JL. Design and analysis of grouprandomized trials: a review of recent methodological developments. Am J Public Health. 2004;94:423-32.

40. NPHS: Australia: The healthiest Country by 2020 - National Preventative Health Strategy - the roadmap for action. Barton: National Preventative Health Taskforce; 2009.

41. Jones RA, Hinkley T, Okely AD, Salmon J. Tracking physical activity and sedentary behavior in childhood: a systematic review. Am J Prev Med. 2013;44(6):651-8.

42. Malina RM. Tracking of physical activity and physical fitness across the lifespan. Res Q Exerc Sport. 1996;67(sup3):S-48-57.

\section{Submit your next manuscript to BioMed Central and we will help you at every step:}

- We accept pre-submission inquiries

- Our selector tool helps you to find the most relevant journal

- We provide round the clock customer support

- Convenient online submission

- Thorough peer review

- Inclusion in PubMed and all major indexing services

- Maximum visibility for your research

Submit your manuscript at www.biomedcentral.com/submit
Biomed Central 